\title{
Best Cover Set Selection with Multi-Criteria Approach in Mission-Critical Surveillance for Wireless Image Sensor Networks
}

\author{
El hadji S. Mamour Diop \\ LIUPPA, University of Pau \\ LANI, Gaston Berger University \\ Email: elhadji.diop@univ-pau.fr
}

\author{
Congduc Pham \\ LIUPPA, University of Pau \\ Pau, France 64013 \\ Email: congduc.pham@univ-pau.fr
}

\author{
Ousmane Thiaré \\ LANI, Gaston Berger University \\ Saint-Louis, Senegal \\ Email: ousmane.thiare@edu.ugb.sn
}

\begin{abstract}
Mission-critical surveillance applications such as intrusion detection or disaster response have vital requirement in QoS. We consider a Wireless Image Sensor Network (WISN) with a scheduling of image sensor node's activity based on the application criticality level. This approach allows sentry nodes capable of detecting intrusions with a higher probability and alerting neighbor nodes as well as activating one of its cover sets for disambiguation purposes for instance. In this paper, we propose a multi-criteria approach to select the suitable cover set for reliable transmission of images in mission-critical applications. The proposed approach takes into account cover set size and energy, topology and routing information that affect the image quality at the sink in a multi-hop transmission network. The results show that our proposal provides low packet loss probability, low latency, and higher visual quality of received images at the Sink.
\end{abstract}

\section{INTRODUCTION}

This article focuses on Wireless Image Sensor Networks (WISN) where sensor nodes are equipped with miniaturized visual cameras. We consider WISN for mission-critical surveillance applications [1], [2], [3], [4], [5], [6], [7], [8], [9] where sensors can be thrown in mass when needed for intrusion detection or disaster relief applications. For example figure 1 shows a image sensor in a rocket-shaped case to be thrown from the air which will always touch ground with the embedded camera set in the right way to start sensing and transmitting images.

Surveillance applications have very specific needs due to their inherently critical nature associated to security . Early surveillance applications involving WSN have been applied to critical infrastructures such as production systems or oil/water pipeline systems [10], [11]. There have also been some propositions for intrusion detection applications [12], [13], [7], [8] but most of these studies focused on coverage and energy optimizations without explicitly having the application's criticality in the control loop which is the main concern in our work. The authors in [14], [15] did consider multimedia sensors but once again, the criticality of a surveillance application is not taken into account. For instance, with image sensors, the higher the capture rate is, the better relevant events could be detected and identified. However, even in the case of very
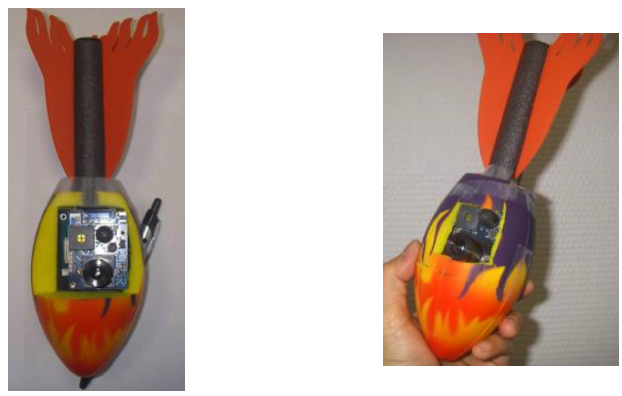

Fig. 1: A video sensor.

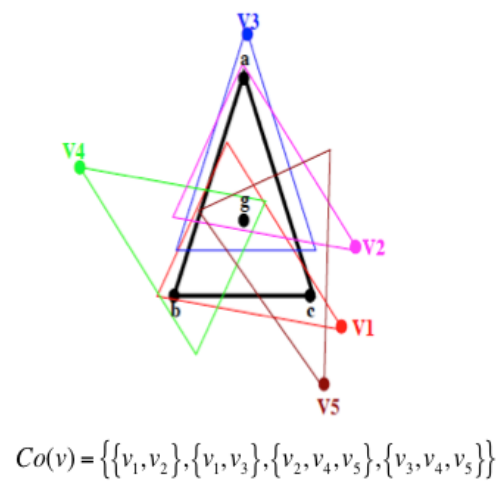

Fig. 2: Coverage model

mission-critical applications, it is not realistic to consider that video nodes should always capture at their maximum rate when in active mode. In randomly deployed sensor networks, provided that the node density is sufficiently high, sensor nodes can be redundant (nodes that monitor the same region) leading to overlaps among the monitored areas. Therefore, a common approach is to define a subset of the deployed nodes to be active while the other nodes can sleep. One obvious way of saving energy is to say that nodes that can be put in sleep mode are typically those whose sensing area are covered by others. In figure 2, the cone of vision of sensor $v$ is represented by the triangle $(a b c)$ and its cover sets are 


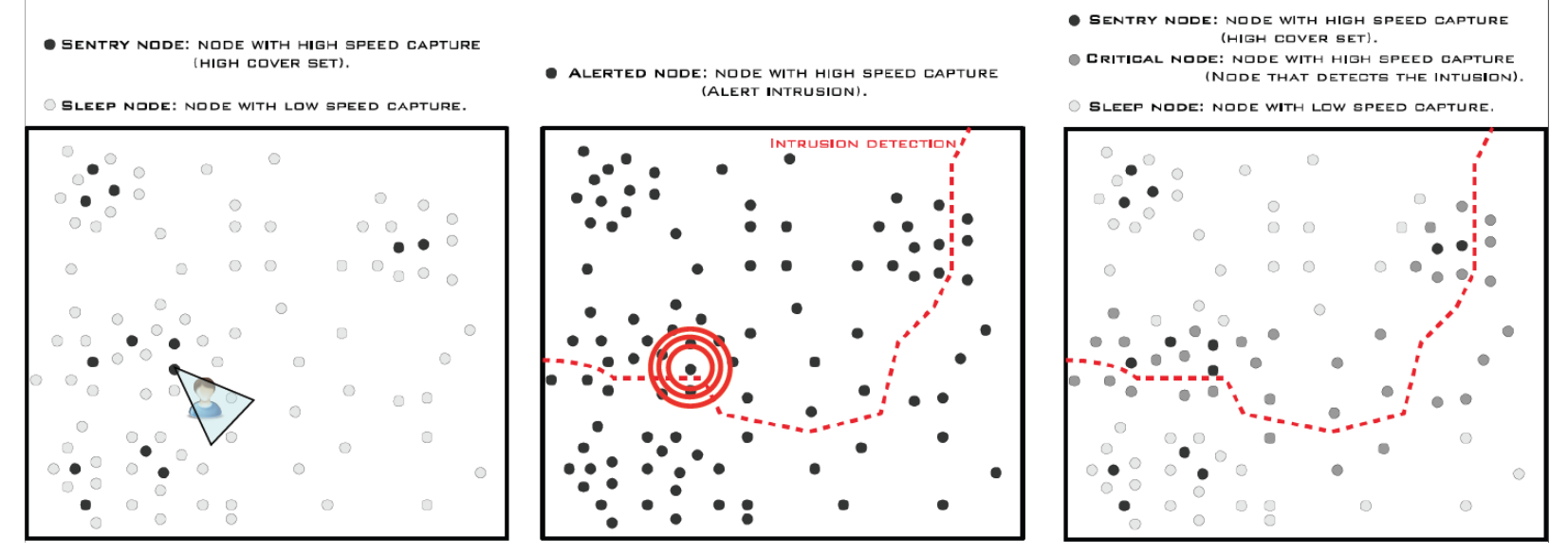

Fig. 3: Wireless Video Sensor Network for intrusion detection.

$C o(v)=\left\{\{v\},\left\{v_{1}, v_{2}\right\},\left\{v_{1}, v_{3}\right\},\left\{v_{2}, v_{4}, v_{5}\right\},\left\{v_{3}, v_{4}, v_{5}\right\}\right\}$. However, in mission-critical applications where some sentry nodes are needed to increase responsiveness, nodes that possess a high redundancy level (their sensing area are covered many times by other nodes so that they have many cover sets) could rather be more active than other nodes with less redundancy level. In [16] the idea we developed is that when a node has several covers, it can increase its frame capture rate because if it runs out of energy it can be replaced by one of its cover sets. Then, depending on the application's criticality, the frame capture rate of those nodes with large number of cover sets can vary: a low criticality level indicates that the application does not require a high image frame capture rate while a high criticality level does.

Figure 3 shows the scenario of a random deployment of visual sensor nodes for a video surveillance application that we address in this paper. Most sensor nodes should move to a so-called hibernate mode in the absence of intrusions: the risk level, noted $r^{0}$, should decrease to $r^{0}=0$ and the sensor nodes should decrease their capture speed (Idle nodes). However, it is also highly desirable that some sensor nodes still keep a relatively high capture speed even when $r^{0}=0$ in order to act as sentry nodes in the surveillance system (figure 3a) to better detect intrusions and to alert, on intrusions, all active nodes to increase their risk level $r^{0}$ to the maximum value, therefore moving to an alerted mode (figure $3 b$ ). This maximum value is the criticality level $R^{0}$ described previously that could be initialized accordingly into all sensor nodes prior to deployment. In this scenario, after some time, an alerted node which does not detect more intrusions, should slowly go back to hibernate mode again by decreasing its risk level $r^{0}$ to 0 in order to save energy, see figure $3 \mathrm{c}$. In this figure, we can also see that an alerted sensor node which does detect an intrusion (all sensor nodes close to the intruder's trajectory dash line - in figure 3c) stays at $r^{0}$ close to the maximum value $R^{0}$.

While in [16], [17] we developed the risk-based scheduling approach and in [18] we compared various methods to build cover sets, the contribution of this paper is at the cover set selection level: when a node $v$ detects an intrusion, it will send one or several images to the sink, alert its neighbor nodes and will activate one of its cover set. On activation, nodes of a cover set will also send one or several images to the sink to provide more information for disambiguation purposes for instance. Obviously, cover sets of a given node have different size, level of coverage/energy, and also different performance level for transferring large amount of data to the sink. In the context of mission-critical application, detecting event is important but receiving images with the highest quality at the sink is also very important. We propose in this article a multi-criteria approach for selecting the best cover set to activate. The proposed approach takes into account various parameters that affect the image quality at the sink in a multihop transmission network.

The remainder of the paper is structured as follows. Section II describes the main guidelines of our proposed multi-criteria approach. We then present in details the various weight definitions used in the multi-criteria approach in Section III. Simulation results are presented in Section IV and we conclude in Section V.

\section{MULTI-CRITERIA APPROACH}

Multi-criteria analysis is a set of methods for aggregating multiple criteria in order to take one or more decisions. Diversity of these methods lies in the way to synthesize information being kept in each criterion. A classification according to three major approaches is suggested: complete, partial and local aggregations [19]. Thanks to its simplicity and its effectiveness, the weighted average, one of the better known techniques of complete aggregation, is used as our score attribution system for cover sets. We give below a definition of this score.

$$
\text { Definition 2.1: Let } C o(v) \text { = }
$$
$\left\{C o_{1}(v), \cdots, C o_{i}(v), \cdots, C o_{n}(v)\right\}$ the set of $n$ cover sets of video sensor $v$ and $M=\left\{M_{1}, \cdots, M_{j}, \cdots, M_{k}\right\}$ a set of $k$ metrics. We define the score of a cover set $C_{i}(v)$, 
denoted $\operatorname{Score}\left(\mathrm{Co}_{i}(v)\right)$, as follows:

$$
\operatorname{Score}\left(C_{i}(v)\right)=\frac{\sum_{j=1}^{k} P_{i . j}}{k}, 1 \leqslant i \leqslant n
$$

where $P_{i . j}$ is value of the metric $M_{j}$ in cover set $C o_{i}(v)$. In WSN, one issue of prime importance is related of energy: scarcity of this resource has a direct impact on cover sets selection and routing strategies. In most considered applications, sensors are densely scattered by aircraft in difficult or dangerous to access areas. Therefore no human intervention is possible and their battery is considered to be the most limiting resource. A cover set consisting of a set of video sensors, we can assign an energy level to it.

Definition 2.2: Let $C_{i}(v)$ be a cover set of video node $v$. We define energy (or lifetime) of $C o_{i}(v)$, denoted $E\left(C o_{i}(v)\right)$, as :

$$
E\left(C o_{i}(v)\right)=\min _{u \in C o_{i}(v)} E(u)
$$

where $E(u)$ is residual energy of video node $u$. Then, our algorithm for selecting the best cover set for a sensor node is essentially based on the following definition.

Definition 2.3: $\operatorname{Co}_{t}(v) \in C o(v)$ is defined as the best cover set of video sensor $v$ iff :

$$
\operatorname{Score}\left(\operatorname{Co}_{t}(v)\right)=\max _{C o_{i}(v) \in C o(v), i \neq t} \operatorname{Score}\left(C_{i}(v)\right)
$$

and

$$
E\left(C o_{t}(v)\right) \geqslant E_{\text {threshold }}
$$

where $E_{\text {threshold }}$ is the minimum required energy threshold of a cover set to participate in the cover set activation process.

\section{DEFINING RELEVANT WEIGHTS FOR THE MULTI-CRITERIA APPROACH}

Our multi-criteria approach can be compared with so-called cross-layer approaches where information from lower levels are used by higher levels (such as the application level). These approaches are widely used in sensor networks [20], [21], but it is necessary to pay particular attention to what information should be considered and to avoid those that are difficult to obtain in a network with a large number of nodes. For example, network and/or link load is a difficult information to estimate, especially in a wireless network where the size of buffer queues is not simply correlated with the network load due to interference phenomena or contention on the radio support [22]. In our proposal, we use parameters such as the number of 1-hop and 2-hop neighbors. These information are readily available at a modest cost. We link this information to the Euclidean distance to the destination. This distance is also easy to get when sensors have either GPS capability or the ability to estimate their position through anchor nodes with GPS capability [23].

In [24], we proposed a scheduling method of sensors that collects for each sensor, during the initialization phase of the network, information about its neighboring nodes. These information are for example GPS position, camera line of sight, angle of view and depth of field of the camera, initial level of criticality, residual energy,... This initialization phase is commonly found in most protocols and corresponds roughly to a neighborhood discovery. This phase requires a message exchange therefore the additional cost is low. Each sensor thus has information on its 1-hop neighborhood. It is then quite easy to get information on 2-hop neighbors, again at a relatively low cost, since each sensor just need to distribute its neighbor table at the end of the first phase. We propose to use this mechanism to determine the selection criteria of cover sets associated with a given criticality level. To do so, each sentry node will identify members of its cover sets (cover members) and retrieve information associated with their neighbor table.

The current version of our approach is based on 6 criteria divided into 2 classes: autonomy and application's criticality.

\section{A. Lifetime}

A cover set of an image sensor node $v$ is defined as a subset of its neighboring nodes which covers its field of view (in [18] we compared various strategies of constructing cover sets). Thus, an important properties for any cover set can be its cardinality, i.e. the cover set size. We therefore use the cover set cardinality as the first criterion $M_{1}$. A cover set containing fewer image sensor nodes has less impact on overall network lifetime. Thus, according to cardinality, the smaller the number of nodes in a cover set, the better it is. Hence the following rule: let $C_{i}(v) \in C o(v)$ and $C o o_{j}(v) \in C o(v), i \neq j$ :

$$
\left|C o_{i}(v)\right|<\left|C o_{j}(v)\right|=>P_{i .1}>P_{j .1}
$$

Since cover set construction methods do not necessarily guarantee their independency, or disjointness, a node can either appear in exactly one cover set or be part of more than one cover set. Here, we choose to limit our study to the intranode dependency, i.e. dependency between cover sets of the same node. It should be noted that this dependency can also exist between cover sets of different nodes. Thus an interesting metric at this level is the degree of influence $M_{2}$ which represents the number of shared nodes with other cover sets of the same node. The activation of a cover set with many shared nodes has a higher impact on the lifetime of other involved cover sets. Therefore the following rule takes this issue into account: let $\mathrm{Co}_{i}(v) \in C o(v)$ and $\mathrm{Co}_{j}(v) \in C o(v), i \neq j$ :

$$
I_{\text {degree }}\left(C o_{i}(v)\right)<I_{\text {degree }}\left(C o_{j}(v)\right)=>P_{i .2}>P_{j .2}
$$

\section{B. Application's criticality}

As said previously, mission-critical applications have QoS requirements such as reliability of received data at the sink, especially for visual information. Congestion and contention on the radio medium are main source of packet losses as the network load increases. Therefore the capture rate of sensor nodes should guide the choice of cover sets as it will have a high impact on the data transmission performance, both at MAC and network level. We thus propose 2 criteria reflecting these requirements. 
1) Reliability: The detection of an event by a sentry can trigger transmission of large amounts of data (image sequences) from multiple sources (cover members of selected cover set) in the network. With no control, this can produce significant data losses due to network congestion. Multipath routing is often regarded as a solution to improve communication performance in WSN: data transmission reliability, bandwidth aggregation, load balanced transmission, congestionfree transmission, low latency transmission,... [25], [26], [27]. Multipath routing is the establishment of multiple paths between a pair (source, destination) for data transmission. To improve reliability, we study the degree of satisfaction of cover sets from a multipath routing perspective by defining two criteria that capture the network topology: 1-hop and 2hop available neighbors (respectively criteria $M_{3}$ and $M_{4}$ ). An eligible neighbor for a cover set is any neighbor of the cover members except (i) the original node that has issued the activation message and (ii) the other cover members. These criteria are controlled by the following weights. Let $C_{i}(v) \in C o(v)$ and $v_{t} \in C o_{i}(v)$ :

$$
P_{i .3}=\sum_{t=1}^{\left|C o_{i}(v)\right|} \frac{\text { Nb1HAvNeighbors }\left(v_{t}\right)}{\text { NbOptimalPaths }\left(v_{t}\right)}
$$

$N b 1 H A v N$ eighbors $\left(v_{t}\right)$ is number of 1-hop available neighbors of node $v_{t}$ and NbOptimalPaths $\left(v_{t}\right)$ the number of optimal paths of $v_{t}$. We define NbOptimalPaths $\left(v_{t}\right)$ to be proportional to its capture rate. Linking the capture rate to the number of required paths to correctly transfer images is an original feature of our approach because capture rates can be very different from a sensor to another since some area parts could be at a higher criticality level than other parts [17]. As scheduling of sensors is very dynamic for these missioncritical applications, the best cover set is highly dependent on the required capture rate.

For criterion $M_{4}$, we calculate the ratio between the number of 2-hop neighbors and the number of 1-hop neighbors of a node $v_{t}$ belonging to cover set $C o_{i}(v)$. Formally, $M_{4}$ is given by the following equation with $v_{t} \in C o_{i}(v)$ :

$$
P_{i .4}=\sum_{t=1}^{\left|C o_{i}(v)\right|} \frac{N b 2 H A v N \text { eighbors }\left(v_{t}\right)}{N b 1 H A v N \text { eighbors }\left(v_{t}\right)}
$$

$\mathrm{Nb2HAvNeighbors}\left(v_{t}\right)$ is the number of 2-hop available neighbors of node $v_{t}$. This criteria probabilistically expresses the fact that 1-hop neighbors (for instance 4 neighbors) may have different paths to go to the Sink. If the number of 2-hop neighbors is also 4 , then this ratio is 1 and there is potentially for each 1-hop neighbor a different path from other 1-hop neighbors to the Sink. If this ratio exceeds 1 , it is even better. However, there is no strict guarantees since a 1-hop neighbor may well have all the 2-hop neighbors of $v_{t}$. Here we find it very difficult to obtain and consider accurate information and this is the reason why we propose a probabilistic approach that has the advantage of being very simple and requiring only a small additional cost in terms of message exchanged. The method we take here is an on-demand method: as all nodes know their 2-hop neighbors, a node $v$ with a cover set $C o_{i}(v)$ would send a request to its cover members to get the list of their 2-hop neighbors.

2) Latency: The image capture rate is particularly crucial for mission-critical surveillance applications. In addition, most of these applications are delay sensitive. Therefore, It is necessary to reduce transmission delays by advocating the use of the shortest path with minimum end-to-end delay transmission [28]. Thus, we evaluate the degree of satisfaction of cover sets for an optimal multipath geographic routing by establishing two other criteria: closest 1-hop and 2-hop available neighbors to the Sink in Euclidean distance (respectively criteria $M_{5}$ and $M_{6}$ ). The weighting system is quite similar to previous equation and we define the following weights:

$$
P_{i .5}=\sum_{t=1}^{\left|C o_{i}(v)\right|} \frac{N b C 1 H \text { AvNeighbors }\left(v_{t}\right)}{\text { NbOptimalPaths }\left(v_{t}\right)}
$$

where $N b C 1 H A v N$ eighbors $\left(v_{t}\right)$ is the number of closest 1hop available neighbors of node $v_{t}$.

$$
P_{i .6}=\sum_{t=1}^{\left|C o_{i}(v)\right|} \frac{N b C 2 H A v N \text { eighbors }\left(v_{t}\right)}{N b C 1 H A v N \text { eighbors }\left(v_{t}\right)}
$$

where $\mathrm{NbC2HAvNeighbors}\left(v_{t}\right)$ is the number of closest 2hop available neighbors of node $v_{t}$.

\section{Simulation Results}

We evaluate our multi-criteria proposition with the Castalia [29] simulator for WSN based on the OMNeT++ [30] simulator. We consider an homogenous WISN where image sensors are randomly deployed in a $75 m * 75 m$ area. Sensors have an $60^{\circ}$ angle of view, a depth of view of $25 \mathrm{~m}$ and a communication range of $30 \mathrm{~m}$. We define different network topologies by varying the number of nodes, hence the node density (see Figure 4). In what follows, we show the performance of our proposal in taking into account topology and dependency information at the routing layer. For each topology, we consider two scenarios for transmission of images by selected cover sets by our approach:

- NO_INFO: no additional information to routing protocol. We use the standard GPSR routing protocol [31].

- WITH_INFO: routing by taking account selection information such as prohibited nodes per cover set (shared nodes, cover members). We used a modified version of GPSR to support multipath transmission.

In addition, we varied the capture rate of cover members from 1 to 3 frames/s. CSMA/CA is used at the MAC layer. We monitor the average packet loss, the average quality of received images at the Sink, and the average image transmission delay to the Sink.

As described in Section I, when a node detects an intrusion it $(i)$ alerts his neighborhood, (ii) sends image(s) and (iii) activates at the same time one of its cover sets which, when activated, also sends image(s) to the Sink. The simulation 


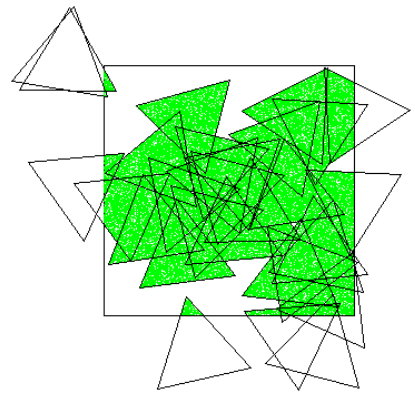

(a) Topology with 30 nodes

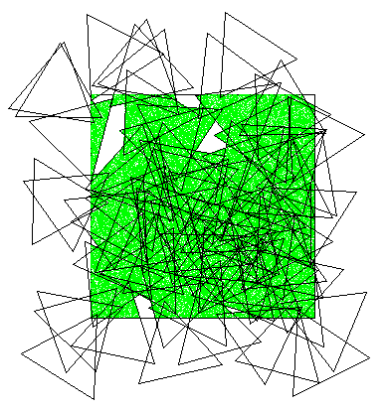

(c) Topology with 80 nodes

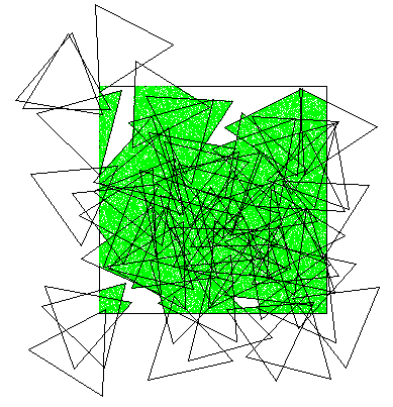

(b) Topology with 60 nodes

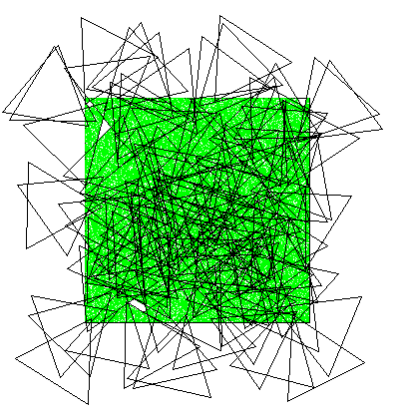

(d) Topology with 110 nodes
Fig. 4: Various network topologies.

model implements the transmission of real image files by taking into account all communication layers. We use a suitable image format for sensor networks that combines robustness with respect to loss, low power consumption for compression and small file size [32], [33]. An image has 320x320 pixels with 256 gray levels for a size of 17.199 bytes. This image is sent in 302 packets to meet the radio constraints of most sensor boards. Energy consumptions have been experimentally measured on Cyclop boards with the image encoding method implemented by the authors of [33].

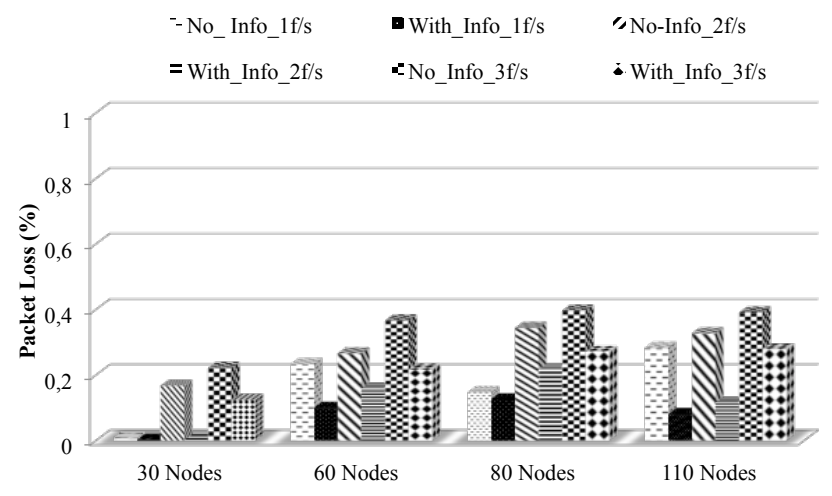

Fig. 5: Average packet loss.

\section{A. Packet loss}

Figure 5 shows that the packet loss can be decreased by taking into account the selection information (WITH_INFO), especially when the capture rate increases. By comparing histograms in pairs (NO_INFO with 1f/s (No_Info_1f/s) and WITH_INFO with 1f/s (With_Info_1f/s),...) this result can be explained by the load balancing and the marking of prohibited nodes which reduces network congestion. We however noticed that packet losses can not be completely avoided, even with WITH_INFO mode. These losses are due to the participation of some nodes in several activated cover sets at the same time. For example if a node $v$ appears in $x$ activated cover sets with capture rate of $y$ frames/s, then $v$ will send $x * y$ frames/s. Further study of inter-cover set dependency is therefore promising and can be studied in future works.

\section{B. Quality of received images}

Reception of a large number of images at the Sink does not necessarily mean that they are all usable. Proportion of packet loss has a direct impact on the quality of the received image. Indeed a received image is either complete (no packet loss) or truncated. According to our observations, a truncated image is not usable (for the end user) if the packet loss rate is above $64 \%$. WITH_INFO mode provides at least $60 \%$ of good quality images (images with less than $64 \%$ of packet losses) as shown in Figure 6.

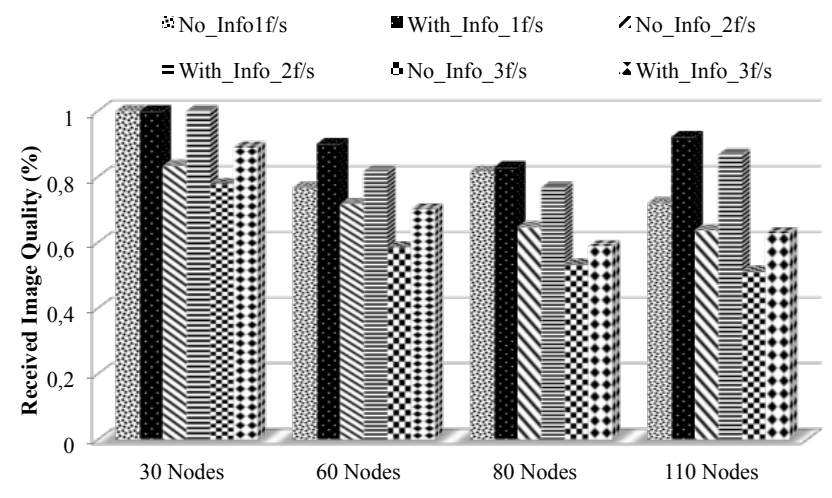

Fig. 6: Average quality of received images.

\section{Image transmission delay}

The number of packet losses has also a direct impact on the image transmission delay. The implemented decoder can display an image regardless of the number of packets received and regardless of their reception order. Nevertheless, a timer is set at the reception of the first image packet and will trigger the display of the image. If the last packet is received at the Sink, the image will be displayed immediately. When the number of packet losses is large, the latency can be as high as the display timer which is set to 10s. With low loss probability, the latency is also low and depends on the number of hops. In our simulations, the latency for receiving a complete image is 1.14s. In WITH_INFO mode, the average transmission delay is smaller than the NO_INFO mode and stays below half the timer value as illustrated in Figure 7. In some cases, the latency is particularly small but this is due to the reception of the last 
image packet when many packets have been lost. If the Sink has high computing power, it can decode and display images in real-time and consider packets as they arrive.

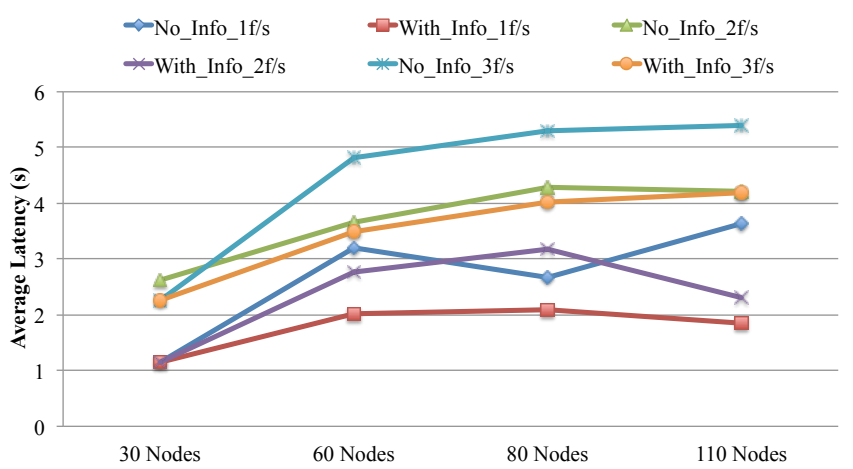

Fig. 7: Average image transmission delay.

\section{CONCLUSION}

In this paper we proposed a multi-criteria method and defined criteria grouped into 2 classes (lifetime and application's criticality). This method is effective to $(i)$ select the suitable cover sets for transmitting images in mission-critical applications, (ii) serve as a base for geographical routing protocol to establish paths according to the application's needs. The advantage of such a multi-criteria approach is the ability to connect several important parameters that depend on the capture rate and the network layer topology. It is also possible to dynamically change the weights according to the application's criticality. We evaluated our proposition by simulation and the results show that a controlled cover set selection approach increases reliability and thereby visual quality of received images at the Sink. These performances are improved by taking account selection information at the routing layer. Future researches in this area are directed towards a better management of shared nodes (those present in several cover sets) to reduce inter-path interference and congestion encountered in multipath routing.

\section{REFERENCES}

[1] H. F. R. Collins, A. Lipton and T. Kanade, "Algorithms for cooperative multisensor surveillance," Proceedings of the IEEE, 89(10), 2001.

[2] T. Yan, T. He, and J. A. Stankovic, "Differentiated surveillance for sensor networks," in SenSys '03: Proceedings of the 1st international conference on Embedded networked sensor systems. New York, NY, USA: ACM, 2003, pp. 51-62.

[3] T. He et al., "Energy-efficient surveillance system using wireless sensor networks," in MobiSys '04: Proceedings of the 2nd international conference on Mobile systems, applications, and services. New York, NY, USA: ACM, 2004, pp. 270-283.

[4] S. Oh, P. Chen, M. Manzo, and S. Sastry, "Instrumenting wireless sensor networks for real-time surveillance," in Proc. of the International Conference on Robotics and Automation, May 2006.

[5] R. Cucchiara et al., "Using a wireless sensor network to enhance video surveillance," J. Ubiquitous Computing and Intelligence, 1(2), 2007.

[6] M. Lu, J. Wu, M. Cardei, and M. Li, "Energy-efficient connected coverage of discrete targets in wireless sensor networks," International Journal of Ad Hoc and Ubiquitous Computing, 4(3-4), pp. 137-147, 2009.
[7] E. Freitas et al., "Evaluation of coordination strategies for heterogeneous sensor networks aiming at surveillance applications," in IEEE Sensors, 2009.

[8] M. Keally, G. Zhou, and G. Xing, "Watchdog: Confident event detection in heterogeneous sensor networks," Real-Time and Embedded Technology and Applications Symposium, IEEE, 2010.

[9] A. . Garcia-Sanchez, F. Garcia-Sanchez, and J. Garcia-Haro, "Wireless sensor network deployment for integrating video-surveillance and datamonitoring in precision agriculture over distributed crops," Computers and Electronics in Agriculture, vol. 75, no. 2, 2011.

[10] L. N. I. Stoianov and S. Madden, "Pipenet: A wireless sensor network for pipeline monitoring." in ACM IPSN, 2007.

[11] S. C. M. Albano and R. D. Pietro, "A model with applications for data survivability in critical infrastructures," Journal of Information Assurance and Security, vol. 4, 2009.

[12] O. Dousse, C. Tavoularis, and P. Thiran, "Delay of intrusion detection in wireless sensor networks," in ACM MobiHoc, 2006.

[13] Y. Zhu and L. M. Ni, "Probabilistic approach to provisioning guaranteed qos for distributed event detection," in IEEE INFOCOM, 2008.

[14] A. Czarlinska and D. Kundur, "Wireless image sensor networks: event acquisition in attack-prone and uncertain environments," Multidimensional Syst. Signal Process., vol. 20, pp. 135-164, Jun. 2009.

[15] M. Alaei and J. M. J. M. Barcelo-Ordinas, "Priority-based node selection and scheduling for wireless multimedia sensor networks," in IEEE WiMob, 2010

[16] A. Makhoul, R. Saadi, and C. Pham, "Risk management in intrusion detection applications with wireless video sensor networks," in IEEE WCNC, 2010.

[17] A. Makhoul, R. Saadi et C. Pham, "Risk-based adaptive scheduling in randomly deployed video sensor networks for critical surveillance applications," Journal of Network and Computer Applications (JNCA), Elsevier, pp. 783-795, 34(2), 2011.

[18] C. Pham et A. Makhoul, "Performance study of multiple cover-set strategies for mission-critical video surveillance with wireless video sensors," IEEE WiMob 2010, Canada, October 2010.

[19] S. B. Mena, "Introduction aux méthodes multicritères d'aide à la décision," Biotechnol. Aron. Soc. Environ. 20004 (2), pp. 83-93, 2000.

[20] I. T. Almalkawi, M. G. Zapata, and J. N. Al-Karaki, "A cross-layer-based clustered multipath routing with qos-aware scheduling for wireless multimedia sensor networks," International Journal of Distributed Sensor Networks, 2012

[21] L. Mendes and J. Rodrigues, "A survey on cross-layer solutions for wireless sensor networks," Journal of Networks and Computer Applications, vol. 34, 2011.

[22] C. Y. Wan and S. B. Eisenman, "Coda: Congestion detection and avoidance in sensor networks," in ACM SenSys, pp. 266-279, 2003.

[23] S. Tanvir, E. Schiller, and A. Duda, "Propagation protocols for networkwide localization based on two-way ranging," in IEEE WCNC, 2010.

[24] A. Makhoul, R. Saadi et C. Pham, "Surveillance vidéo sur réseaux de capteurs sans fils : ordonnancement adaptatif avec prise en compte de la criticité," 14e CFIP, Strasbourg, France, Octobre 2009.

[25] S. Guo et T. D. C. Little, "A video delivery in wireless sensor networks," Streaming Media Architectures, Techniques, and Applications: Recent Advances. IGI Global, pp. 239-260, 2011.

[26] S. Soro, W. Heinzelman, "A survey of visual sensor networks," Advances in Multimedia, vol. 2009, no. ID 640386, p. 21, May 2009.

[27] M. Radi, B. Dezfouli, K.A. Bakar et M. Lee, "Multipath routing in wireless sensor networks : Survey and research challenges," Sensors, no. $650-685,2012$.

[28] S. Medjiah, T. Ahmed, F. Krief et P. Gélard, "Agem: un protocole de routage géographique angulaire adaptatif," $14 e$ CFIP, Strasbourg, France, Octobre 2009.

[29] [Online]. Available: http://castalia.npc.nicta.com.au/

[30] [Online]. Available: http://www.omnetpp.org/

[31] B. Karp, H. T. Kung, "Gpsr: Greedy perimeter stateless routing for wireless networks," ACM, MobiCom, 2000.

[32] C. Duran-Faundez, V. Lecuire, and F. Lepage, "Tiny block-size coding for energy-efficient image compression and communication in wireless camera sensor networks," Signal Processing: Image Communication, vol. 26, pp. 466-481, 2011.

[33] V. Lecuire, L. Makkaoui, and J.-M. Moureaux, "Fast zonal dct for energy conservation in wireless image sensor networks," Electronics Letters, vol. 48, 2012. 